\title{
Publisher Correction: Carrier lifetime enhancement in halide perovskite via remote epitaxy
}

Jie Jiang (1) 1,2,9, Xin Sun (10) 3,9, Xinchun Chen (1) ${ }^{4}$, Baiwei Wang (1) 2, Zhizhong Chen², Yang Hu ${ }^{2}$, Yuwei Guo²,

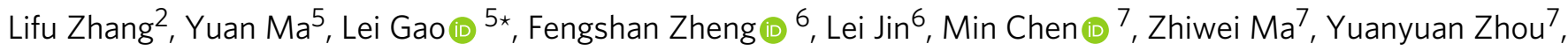
Nitin P. Padture (1) ${ }^{7}$, Kory Beach (10) ${ }^{3}$, Humberto Terrones (10 ${ }^{3}$, Yunfeng Shi ${ }^{2}$, Daniel Gall (1) ${ }^{2}$, Toh-Ming Lu ${ }^{3}$, Esther Wertz (iD ${ }^{3}$, Jing Feng ${ }^{1 \star} \&$ Jian Shi (iD) ${ }^{2,8 \star}$

Correction to: Nature Communications https://doi.org/10.1038/s41467-019-12056-1, published online 12 September 2019.

The original version of this Article contained an error in the author affiliations. The fifth affiliation incorrectly read 'Beijing Advanced Innovation Center for Materials Genome Engineering, Institute for Advanced Materials and Technology, University of Science and Technology, Beijing 100083, China.' The correct version reads 'Beijing Advanced Innovation Center for Materials Genome Engineering, Institute for Advanced Materials and Technology, University of Science and Technology Beijing, Beijing 100083, China.' This has now been corrected in both the PDF and HTML versions of the Article.

Published online: 16 October 2019

Open Access This article is licensed under a Creative Commons Attribution 4.0 International License, which permits use, sharing, adaptation, distribution and reproduction in any medium or format, as long as you give appropriate credit to the original author(s) and the source, provide a link to the Creative Commons license, and indicate if changes were made. The images or other third party material in this article are included in the article's Creative Commons license, unless indicated otherwise in a credit line to the material. If material is not included in the article's Creative Commons license and your intended use is not permitted by statutory regulation or exceeds the permitted use, you will need to obtain permission directly from the copyright holder. To view a copy of this license, visit http://creativecommons.org/licenses/by/4.0/.
}

(C) The Author(s) 2019

\footnotetext{
${ }^{1}$ Department of Materials Science and Engineering, Kunming University of Science and Technology, Kunming, Yunnan 650093, China. ${ }^{2}$ Department of Materials Science and Engineering, Rensselaer Polytechnic Institute, Troy 12180, USA. ${ }^{3}$ Department of Physics, Applied Physics and Astronomy, Rensselaer Polytechnic Institute, Troy, NY 12180, USA. ${ }^{4}$ State Key Laboratory of Tribology, Tsinghua University, Beijing 100084, China. ${ }^{5}$ Beijing Advanced Innovation Center for Materials Genome Engineering, Institute for Advanced Materials and Technology, University of Science and Technology Beijing, Beijing 100083, China. ${ }^{6}$ Ernst Ruska-Centre for Microscopy and Spectroscopy with Electrons and Peter Grünberg Institute, Forschungszentrum Jülich, Jülich, Germany. 7 School of Engineering, Brown University, Providence, RI 02912, USA. ${ }^{8}$ Center for Materials, Devices, and Integrated Systems, Rensselaer Polytechnic Institute, Troy, NY 12180, USA. ${ }^{9}$ These authors contributed equally: Jie Jiang, Xin Sun. *email: gaolei@ustb.edu.cn; vdmzsfj@qq.com; shij4@@rpi.edu
} 